\title{
A new analytical modelling for fractional telegraph equation via Elzaki transform
}

\section{Huan Li}

College of Mathematics and Information Science, Henan polytechnic University, Jiaozuo 454000, China

\begin{abstract}
The main aim of this paper is to propose a new and simple algorithm for space-fractional telegraph equation, namely new fractional homotopy analysis transform method (FHATM). The fractional homotopy analysis transform method is an innovative adjustment in Elzaki transform algorithm (ETA) and makes the calculation much simpler. The numerical solutions obtained by proposed method indicate that the approach is easy to implement and computationally very attractive. Finally, several numerical examples are given to illustrate the accuracy and stability of this method.
\end{abstract}

Keywords: Fractional telegraph equation; Elzaki transform method; fractional homotopy analysis transform method (FHATM);

\section{Council for Innovative Research}

Peer Review Research Publishing System

Journal: JOURNAL OF ADVANCES IN MATHEMATICS

Vol.11, No.9

www.cirjam.com, editorjam@gmail.com 


\section{INTRODUCTION}

In rencent years, fractional calculus found many applications. Some fractional partial differential equations were successfully used in various field of physical sciences. For example, Caputo[1], Giona and Roman[2], Hilfer[3], Mainardi and Tomirotti[4] et al.

The diffrernt type solutions of the fractional telegraph equations have been discussed recently by several authors. For example Momani[5] by using decomposition method, Yildirim[6] by homotopy perturbation method, chen[7] et al. by the method of separable variables, Huang[8] by Cauchy problem, Biazar and Eslami[9] by using diffrerntial transform method, Sunil[10] by using Laplace transform. Our concern in this paper is to consider the space-fractional telegraph equations as

$$
D_{x}^{2 \alpha} u(x, t)=D_{t}^{2} u(x, t)+a D_{t}(x, t)+b u^{n}(x, t)+f(x, t), \quad 0<\alpha \leq 1,
$$

where $a, b$ and $n$ are given constants, $f(x, t)$ is given function.

In this paper, the homotopy analysis transform method (HATM) basically illustrates how the Elzaki transform can be used to approximate the solutions of the linear and nonlinear partial differential equation by manipulating the homotopy analysis method. The proposed method is coupling of the homotopy analysis method and Elzaki transform. Homotopy analysis method (HAM) was first proposed and applied by Liao[11, 12, 13, 14] based on homotopy, a fundamental concept in topology and differential geometry.

The main purpose of this article is introduce a new analytical and approximate solution of space-fractional telegraph equation by means of fractional homotopy analysis transform method, which is coupling of homotopy analysis method and Elzaki transform method.

\section{Preliminaries}

\subsection{Fractional calculus}

We recall some definitions of fractional derivatives and fractional integrals. Let $\Gamma(\cdot)$ denote the Gamma function. For any positive integer $n$ and $n-1 \leq \gamma<n$, the Caputo derivative and the Riemann"CLiouville derivative of order $\gamma$ are defined, respectively, as follows.

- Caputo derivative

$$
{ }^{C} D_{a^{+}}^{\gamma} v(x)=\frac{1}{\Gamma(n-\gamma)} \int_{a}^{x} \frac{v^{(n)}(t)}{(x-t)^{\gamma-n+1}} d t, \quad a \leq x \leq b, n-1 \leq \gamma<n .
$$

where $v^{(n)}(t)=\frac{d^{n} v(t)}{d t^{n}}$

- Riemann-Liouville dervative

$$
{ }^{R} D_{a^{+}}^{\gamma} v(x)=\frac{1}{\Gamma(n-\gamma)} \frac{d^{n}}{d x^{n}} \int_{a}^{x} \frac{v(t)}{(x-t)^{\gamma-n+1}} d t, \quad a \leq x \leq b, n-1 \leq \gamma<n .
$$

\subsection{Basic definition of fractional calculus}

This section, we give some basic definitions and properties:

Definition 2.1 We Consider function in the set $A$, defined by[15]:

$$
A=\left\{f(t): \exists M, k_{1}, k_{2}>0,|f(t)|<M e^{\frac{|t|}{k_{j}}}, \text { if } t \in(-1)^{j} \mathbf{X}[0, \infty)\right\},
$$

For a given function in the set $A$, the constant $\mathrm{M}$ must be finite number, $k_{1}, k_{2}$ may be finite or infinite.

The Elzaki transform denoted by the operator $E($.$) defined by the integral equations$

$$
T(v)=E[f(t)]=v \int_{v}^{\infty} e^{\frac{-t}{v}} f(t) d t, v \in\left[-k_{1}, k_{2}\right]
$$

Definition 2.2 The Elzaki transform of $f(t)=t^{\alpha}$ is defined as[16]: 


$$
E\left[t^{\alpha}\right]=v \int_{0}^{\infty} e^{\frac{-t}{v}} t^{\alpha} d t=v^{\alpha+2} \Gamma(\alpha+1) .
$$

Definition 2.3 The Elzaki transform $E[f(t)]$ of the Riemann"CLiouville fractional is defined as[16]:

$$
E\left[I^{\alpha} f(t)\right]=v^{\alpha+1} T(v) .
$$

Definition 2.4 The Elzaki transform $E[f(t)]$ of the caputo fractional is defined as[16]:

$$
E\left[D_{x}^{n \alpha} u(x, t)\right]=\frac{T(v)}{v^{n \alpha}}-\sum_{k=0}^{n-1} s^{2-n \alpha+k} u^{k}(0, t), n-1<n \alpha \leq n .
$$

\section{Basic idea of newly fractional homotopy analysis transform method (FHATM)}

We consider the following fractional partial differential equation as:

$$
D_{t}^{n \alpha} u(r, t)+R[r] u(r, t)+N[r] u(r, t)=g(r, t), t>0, r \in R^{3}, n-1<n \alpha \leq n,
$$

where $D_{t}^{n \alpha}$ is Caputo differential coefficient, $R[r]$ is the linear operator for $r \in R^{3}, N[r]$ is the general nonlinear operator for $r \in R^{3}$ and $g(r, t)$ are continuous functions. For simplicity, we ignore all initial and boundary conditions, which can be treated in similar way. Now the methodology consists of applying Elzaki transform first on both sides of equation(7), we get

$$
E\left[D_{t}^{n \alpha} u(r, t)\right]+E[R[r] u(r, t)+N[r] u(r, t)]=E[g(r, t)] .
$$

According to the differentiation property of the Elzaki transform, we have

$$
E[u(r, t)]-v^{n \alpha} \sum_{k=0}^{n-1} v^{(2-n \alpha+k)} u^{k}(r, 0)+v^{n \alpha} E(R[r] u(r, t)+N[r] u(r, t)-g(r, t))=0 .
$$

We define the nonlinear operator[32]

$$
\begin{aligned}
N[\phi(r, t ; q)]= & E[\phi(r, t ; q)]-v^{n \alpha} \sum_{k=0}^{n-1} v^{(2-n \alpha+k)} u^{k}(r, 0)+v^{n \alpha} E(R[r] \phi(r, t ; q) \\
& +N[r] \phi(r, t ; q)-g(r, t ; q)),
\end{aligned}
$$

where $q \in[0,1]$ be an embedding parameter and $\phi(r, t ; q)$ is the real function of $r, t$ and $q$.

We define the zero order deformation equation

$$
(1-q) \mathbf{L}\left[\phi(r, t ; q)-u_{0}(r, t)\right]=h q H(r, t) N[\phi(r, t ; q)],
$$

where $h$ is a nonzero auxiliary parameter, $H(r, t) \neq 0$ is an auxiliary function, $u_{0}(r, t)$ is an initial guess of $u(r, t)$ and $\phi(r, t ; q)$ is an unknown function.Obviously, when $q=0$ and $q=1$, it hold

$$
\phi(r, t ; 0)=u_{0}(x, t), \phi(r, t ; 1)=u(x, t) .
$$

Thus, as $q$ increases from 0 to 1 , the solution varies from the initial guess $u_{0}(r, t)$ to the solution $u(r, t)$. Expanding $\phi(r, t ; q)$ in Taylori's series with respect to $q$, we have

$$
\phi(r, t ; q)=u_{0}(r, t)+\sum_{m=1}^{\infty} u_{m}(r, t) q^{m}
$$

where

$$
u_{m}(r, t)=\left.\frac{1}{m !} \frac{\partial^{m} \phi(r, t ; q)}{\partial q^{m}}\right|_{q=0} .
$$


If the auxiliary linear operator $\mathbf{L}$, the initial guess $u_{0}(x, t)$ and the auxiliary parameter $h$ and the auxiliary function are properly chosen, the series $(11)$ converges at $q=1$, we have

$$
u(r, t)=u_{0}(r, t)+\sum_{m=1}^{\infty} u_{m}(r, t),
$$

which must be one of the solutions of original nonlinear equations. Define the vectors

$$
\overleftarrow{u}_{n}(r, t)=\left\{u_{0}(r, t), u_{1}(r, t), \cdots u_{n}(r, t)\right\} \text {. }
$$

Define the $m t h$ order deformation equation

$$
\mathbf{L}\left[u_{m}(r, t)-\chi_{m} u_{m-1}(r, t)\right]=h q H(r, t) R_{m}\left(\overleftarrow{u}_{m-1}(r, t)\right) .
$$

Operating the inverse Elzaki transform on both sides of the equation(14), we get

$$
u_{m}(r, t)=\chi_{m} u_{m-1}(r, t)+h q \mathbf{L}^{-1}\left[H(r, t) R_{m}\left(\overleftarrow{u_{m}}\right)(r, t)\right]
$$

where

$$
R_{m}\left(\overleftarrow{u}_{m}(r, t)\right)=\left.\frac{1}{(m-1) !} \frac{\partial^{m-1} \phi(r, t ; q)}{\partial q^{m-1}}\right|_{q=0}
$$

\section{Illustrative examples}

In this section three examples on fractional homogeneous and non-homogeneous space fractional telegraph equations are solved to demonstrate the performance and efficiency of the HAM with new coupling of Elzaki transform method.

Example 4.1 We consider the following homogeneous space-fractional telegraph equation as

$$
\left\{\begin{array}{c}
\frac{\partial^{\alpha} u}{\partial x^{\alpha}}=\frac{\partial^{2} u}{\partial t^{2}}+\frac{\partial u}{\partial t}+u, t \geq 0,0<\alpha \leq 2 \\
u(0, t)=e^{-t}, t \geq 0 \\
\frac{\partial u(0, t)}{\partial x}=e^{-t}, t \geq 0, \\
u(x, 0)=e^{x}, 0<x<1 .
\end{array}\right.
$$

Operating the Elzaki transform on both sides in (16) and after using the differentiation property of Elzaki transform for fractional derivative, we get

$$
\frac{E[u(x, t)]}{v^{\alpha}}-v^{2-\alpha} u(0, t)-E\left[D_{t}^{2} u+D_{t} u+u\right]=0 .
$$

We now define a nonlinear operator as

$$
N[\phi(x, t ; q)]=E[\phi(x, t ; q)]-v^{2} e^{-t}-v^{\alpha} E\left[D_{t}^{2} \phi(x, t ; q)+D_{t} \phi(x, t ; q)+\phi(x, t ; q)\right] .
$$

Using above definition, with assumption $H(x, t)=1$, we construct the zeroth order deformation equation

$$
(1-q) L\left[\phi(x, t ; q)-u_{0}(x, t)\right]=q h N[\phi(x, t ; q)] .
$$

Obviously, when $q=0$ and $q=1$, 


$$
\phi(x, t ; 0)=u_{0}(x, t), \phi(x, t ; 1)=u(x, t) .
$$

Thus, we obtain the $m t h$ order deformation equation

$$
E\left[u_{m}(x, t)-\chi_{m} u_{m-1}(x, t)\right]=h R_{m}\left(\overleftarrow{u}_{m-1}(x, t)\right) .
$$

Operating the inverse Elzaki transform on both sides in (17), we get

$$
u_{m}(x, t)=\chi_{m} u_{m-1}(x, t)+h q E^{-1}\left[R_{m}\left(\tilde{u}_{m}\right)(x, t)\right],
$$

where

$$
R_{m}\left(\overleftarrow{u}_{m-1}(x, t)\right)=E\left[u_{m-1}\right]-\left(1-\chi_{m}\right) v^{2} e^{-t}-v^{\alpha} E\left[D_{t}^{2} u_{m-1}+D_{t} u_{m-1}+u_{m-1}\right] .
$$

Now the solution of equation(18)

$$
u_{m}(x, t)=\left(\chi_{m}+h\right) u_{m-1}-h\left(1-\chi_{m}\right) e^{-t}-h E^{-1}\left(v^{\alpha} E\left[D_{t}^{2} u_{m-1}+D_{-t} u_{m-1}+u_{m-1}\right]\right) .
$$

We choose the initial condition

$$
u_{0}(x, t)=u(0, t)+x u_{x}(0, t)=(1+x) e^{-t}
$$

then, we have

$$
\begin{aligned}
u_{1}= & -h e^{-t}\left(\frac{x^{\alpha}}{\Gamma(\alpha+1)}+\frac{x^{\alpha+1}}{\Gamma(\alpha+2)}\right) \\
u_{2}= & -\frac{h(1+h) e^{-t} x^{\alpha}}{\Gamma(\alpha+1)}-\frac{h(1+h) e^{-t} x^{\alpha+1}}{\Gamma(\alpha+2)}+\frac{h^{2} e^{-t} x^{2 \alpha}}{\Gamma(2 \alpha+1)}+\frac{h^{2} e^{-t} x^{2 \alpha+1}}{\Gamma(2 \alpha+2)}, \\
u_{3}= & -\frac{h(1+h)^{2} e^{-t} x^{\alpha}}{\Gamma(\alpha+1)}-\frac{h(1+h)^{2} e^{-t} x^{\alpha+1}}{\Gamma(\alpha+2)}+\frac{2 h^{2}(1+h) e^{-t} x^{2 \alpha}}{\Gamma(2 \alpha+1)}+\frac{2 h^{2}(1+h) e^{-t} x^{2 \alpha+1}}{\Gamma(2 \alpha+2)} \\
& -\frac{h^{3} e^{-t} x^{3 \alpha}}{\Gamma(3 \alpha+1)}-\frac{h^{3} e^{-t} x^{3 \alpha+1}}{\Gamma(3 \alpha+2)}, \\
= & -\frac{h(1+h)^{3} e^{-t} x^{\alpha}}{\Gamma(\alpha+1)}+\frac{h(1+h)^{3} e^{-t} x^{\alpha+1}}{\Gamma(\alpha+2)}+\frac{3 h^{2}(1+h)^{2} e^{-t} x^{2 \alpha}}{\Gamma(2 \alpha+1)}+\frac{x^{2 \alpha+1}}{\Gamma(2 \alpha+2)} \\
\left.u_{4}\right) & -\frac{3 h^{3}(1+h) e^{-t} x^{3 \alpha}}{\Gamma(3 \alpha+1)}+\frac{3 h^{3}(1+h) e^{-t} x^{3 \alpha+1}}{\Gamma(3 \alpha+2)}+\frac{h^{4} e^{-t} x^{4 \alpha}}{\Gamma(4 \alpha+1)}+\frac{h^{4} e^{-t} x^{4 \alpha+1}}{\Gamma(4 \alpha+2)},
\end{aligned}
$$

If we choose auxiliary parameters $h=-1$, we get the approximate analytical solution of equation (16)

$$
\begin{aligned}
u(x, t)= & e^{-t}\left(1+x+\frac{x^{\alpha}}{\Gamma(\alpha+1)}+\frac{x^{\alpha}}{\Gamma(\alpha+2)}+\frac{x^{2 \alpha}}{\Gamma(2 \alpha+1)}+\frac{x^{2 \alpha}}{\Gamma(2 \alpha+2)}+\cdots\right. \\
& \left.+\frac{x^{n \alpha}}{\Gamma(n \alpha+1)}+\frac{x^{n \alpha}}{\Gamma(n \alpha+2)}+\cdots\right),
\end{aligned}
$$

when we choose $\alpha=2$, equation (16) is a homogeneous space telegraph equation, and the exact solution $u(x, t)=e^{x-t}$. we applied Elzaki transformation and the homotopy analysis method to get the solution which is an exact solution of the standard telegraph equation(16).

Figure 1: when $\alpha=2, \mathrm{x}=1$, the exact solution of example(4.1) and Elzaki transformation

Example 4.2 We consider the following homogeneous space-fractional telegraph equation as[33] 


$$
\left\{\begin{array}{c}
\frac{\partial^{\alpha} u}{\partial x^{\alpha}}=\frac{\partial^{2} u}{\partial t^{2}}+4 \frac{\partial u}{\partial t}+4 u, t \geq 0,0<\alpha \leq 2, \\
u(0, t)=1+e^{-2 t} \\
u_{x}(0, t)=2 \\
u(x, 0)=1+e^{2 x}, 0<x<1 \\
u_{t}(x, 0)=-2 .
\end{array}\right.
$$

Operating the Elzaki transform on both sides in (22) and after using the differentiation property of Elzaki transform for fractional derivative, we get

$$
\frac{E[u(x, t)]}{v^{\alpha}}-v^{2-\alpha} u(0, t)-E\left[D_{t}^{2} u+4 D_{t} u+4 u\right]=0 .
$$

We now define a nonlinear operator as

$$
N[\phi(x, t ; q)]=E[\phi(x, t ; q)]-\left(e^{-2 t}+1\right) v^{2}-v^{\alpha} E\left[D_{t}^{2} \phi(x, t ; q)+4 D_{t} \phi(x, t ; q)+4 \phi(x, t ; q)\right] .
$$

Using above definition, with assumption $H(x, t)=1$, we construct the zeroth order deformation equation

$$
(1-q) \mathbf{L}\left[\phi(x, t ; q)-u_{0}(x, t)\right]=q h N[\phi(x, t ; q)] .
$$

Obviously, when $q=0$ and $q=1$,

$$
\phi(x, t ; 0)=u_{0}(x, t), \phi(x, t ; 1)=u(x, t) .
$$

Thus, we obtain the $m t h$ order deformation equation

$$
\mathbf{L}\left[u_{m}(x, t)-\chi_{m} u_{m-1}(x, t)\right]=h R_{m}\left(\overleftarrow{u}_{m-1}(x, t)\right) .
$$

Operating the inverse Elzaki transform on both sides in (23), we get

$$
u_{m}(x, t)=\chi_{m} u_{m-1}(x, t)+h q \mathbf{L}^{-1}\left[R_{m}\left(\overleftarrow{u_{m}}\right)(x, t)\right],
$$

where

$$
R_{m}\left(\overleftarrow{u}_{m-1}\right)=E\left[u_{m-1}\right]-\left(1-\chi_{m}\right)\left(e^{-2 t}+1\right) v^{2}-v^{\alpha} E\left[D_{t}^{2} u_{m-1}+4 D_{t} u_{m-1}+4 u_{m-1}\right] .
$$

Now the solution of Equation(24)

$$
u_{m}=\left(\chi_{m}+h\right) u_{m-1}-h\left(1-\chi_{m}\right)\left(e^{-2 t}+1\right)-h E^{-1}\left(v^{\alpha} E\left[D_{t}^{2} u_{m-1}+4 D_{-t} u_{m-1}+4 u_{m-1}\right]\right) \text {. }
$$

We choose the initial condition

$$
u_{0}(x, t)=u(x, 0)+x u_{x}(x, 0)=e^{-2 t}+2 x+1,
$$

we get 


$$
\begin{aligned}
u_{1}(x, t)= & -4 h\left(\frac{2 x^{\alpha+1}}{\Gamma(\alpha+2)}+\frac{x^{\alpha}}{\Gamma(\alpha+1)}\right) \\
u_{2}(x, t)= & -\frac{4 h(1+h) x^{\alpha}}{\Gamma(\alpha+1)}-\frac{8 h(1+h) x^{\alpha+1}}{\Gamma(\alpha+2)}+\frac{16 h^{2} x^{2 \alpha}}{\Gamma(2 \alpha+1)}+\frac{32 h^{2} x^{2 \alpha+1}}{\Gamma(2 \alpha+2)}, \\
u_{3}(x, t)= & -\frac{4 h(1+h)^{2} x^{\alpha}}{\Gamma(\alpha+1)}-\frac{8 h(1+h)^{2} x^{\alpha+1}}{\Gamma(\alpha+2)}+\frac{16 h^{2}(1+h) x^{2 \alpha}}{\Gamma(2 \alpha+1)}+\frac{32 h^{2}(1+h) x^{2 \alpha+1}}{\Gamma(2 \alpha+2)} \\
& -\frac{64 h^{3} x^{3 \alpha}}{\Gamma(3 \alpha+1)}-\frac{128 h^{3} x^{3 \alpha+1}}{\Gamma(3 \alpha+2)}, \\
u_{4}(x, t)= & -\frac{4 h(1+h)^{3} x^{\alpha}}{\Gamma(\alpha+1)}-\frac{8 h(1+h)^{3} x^{\alpha+1}}{\Gamma(\alpha+2)}+\frac{16 h^{2}(1+h)^{2} x^{2 \alpha}}{\Gamma(2 \alpha+1)}+\frac{32 h^{2}(1+h)^{2} x^{2 \alpha+1}}{\Gamma(2 \alpha+2)} \\
& -\frac{64 h^{3}(1+h) x^{3 \alpha}}{\Gamma(3 \alpha+1)}-\frac{128 h^{3}(1+h) x^{3 \alpha+1}}{\Gamma(3 \alpha+2)}+\frac{256 h^{4} x^{4 \alpha}}{\Gamma(4 \alpha+1)}+\frac{512 h^{4} x^{4 \alpha+1}}{\Gamma(4 \alpha+2)},
\end{aligned}
$$

If we choose auxiliary parameters $h=-1$, we get the approximate analytical solution of equation(22)

$$
u(x, t)=e^{-2 t}+\left(1+2 x+\frac{4 x^{\alpha}}{\Gamma(\alpha+1)}+\frac{8 x^{\alpha+1}}{\Gamma(\alpha+2)}+\cdots+\frac{2^{2 n} x^{n \alpha}}{\Gamma(n \alpha+1)}+\frac{2^{2 n+1} x^{n \alpha+1}}{\Gamma(n \alpha+2)}+\cdots\right)
$$

Plug $\alpha=2$ into (27), we get

$$
u(x, t)=e^{-2 t}+\left(1+2 x+\frac{(2 x)^{2}}{2 !}+\frac{(2 x)^{3}}{3 !}+\frac{(2 x)^{4}}{4 !}+\cdots+\frac{(2 x)^{n}}{n !}+\cdots\right)
$$

when we choose $\alpha=2$, equation (22) is a homogeneous space telegraph equation, and the exact solution $u(x, t)=e^{x-t}$. we applied Elzaki transformation and the homotopy analysis method to get the solution which is an exact solution of the standard telegraph equation (22).

Example 4.3 We consider the following nonhomogeneous space-fractional telegraph equation as

$$
\left\{\begin{array}{c}
\frac{\partial^{\alpha} u}{\partial x^{\alpha}}=\frac{\partial^{2} u}{\partial t^{2}}+\frac{\partial u}{\partial t}+u-x^{2}-t+1, t \geq 0,0<\alpha \leq 2 \\
u(0, t)=t, t \geq 0 \\
\frac{\partial u(0, t)}{\partial x}=0, t \geq 0 \\
u(x, 0)=x^{2}, 0<x<1
\end{array}\right.
$$

Operating the Elzaki transform on both sides in (29) and after using the differentiation property of Elzaki transform for fractional derivative, we get

$$
\frac{E[u(x, t)]}{v^{\alpha}}-v^{2-\alpha} u(0, t)-E\left[D_{t}^{2} u+D_{t} u+u-x^{2}-t+1\right]=0 .
$$

Now we define a nonlinear operator as

$$
N[\phi(x, t ; q)]=E[\phi(x, t ; q)]-v^{2} t-v^{\alpha} E\left[D_{t}^{2} \phi(x, t ; q)+D_{t} \phi(x, t ; q)+\phi(x, t ; q)-x^{2}-t+1\right] .
$$

Using above definition, with assumption $H(x, t)=1$, we construct the zeroth order deformation equation

$$
(1-q) \mathbf{L}\left[\phi(x, t ; q)-u_{0}(x, t)\right]=q h N[\phi(x, t ; q)] .
$$

Obviously, when $q=0$ and $q=1$, 


$$
\phi(x, t ; 0)=u_{0}(x, t), \phi(x, t ; 1)=u(x, t) .
$$

Thus, we obtain the $m t h$ order deformation equation

$$
\mathbf{L}\left[u_{m}(x, t)-\chi_{m} u_{m-1}(x, t)\right]=h R_{m}\left(\overleftarrow{u}_{m-1}(x, t)\right) .
$$

Operating the inverse Elzaki transform on both sides in (30), we get

$$
u_{m}(x, t)=\chi_{m} u_{m-1}(x, t)+h q E^{-1}\left[R_{m}\left(\tilde{u}_{m}\right)(x, t)\right],
$$

where

$$
R_{m}\left(\overleftarrow{u}_{m-1}\right)=E\left[u_{m-1}\right]-\left(1-\chi_{m}\right) t v^{2}-v^{\alpha} E\left[D_{t}^{2} u_{m-1}+D_{t} u_{m-1}+u_{m-1}-x^{2}-t+1\right] .
$$

Now the solution of Equation(31)

$$
u_{m}=\left(\chi_{m}+h\right) u_{m-1}-h\left(1-\chi_{m}\right) t-h E^{-1}\left(v^{\alpha} E\left[D_{t}^{2} u_{m-1}+D_{-t} u_{m-1}+u_{m-1}-x^{2}-t+1\right]\right) \text {. }
$$

We choose the initial condition

$$
u_{0}(x, t)=u(0, t)+x u_{x}(0, t)=t
$$

After calculation£ $\neg$ we get

$$
\begin{aligned}
u_{1}(x, t)= & -\frac{2 h x^{\alpha}}{\Gamma(\alpha+1)}+\frac{2 h x^{\alpha+1}}{\Gamma(\alpha+3)}, \\
u_{2}(x, t)= & -\frac{2 h(1+h) x^{\alpha}}{\Gamma(\alpha+1)}+\frac{2 h(1+h) x^{\alpha+2}}{\Gamma(\alpha+3)}+\frac{2 h^{2} x^{2 \alpha}}{\Gamma(2 \alpha+1)}-\frac{2 h^{2} x^{2 \alpha+2}}{\Gamma(2 \alpha+3)} \\
& +\frac{2 h x^{\alpha+2}}{\Gamma(\alpha+3)}-\frac{(t-1) h x^{\alpha}}{\Gamma(\alpha+1)}, \\
u_{3}(x, t)= & -\frac{2 h(1+h)^{2} x^{\alpha}}{\Gamma(\alpha+1)}+\frac{2 h(1+h)^{2} x^{\alpha+2}}{\Gamma(\alpha+3)}+\frac{4 h^{2}(1+h) x^{2 \alpha}}{\Gamma(2 \alpha+1)}-\frac{4 h^{2}(1+h) x^{2 \alpha+2}}{\Gamma(2 \alpha+3)} \\
& -\frac{2 h^{3} x^{3 \alpha}}{\Gamma(3 \alpha+1)}+\frac{2 h^{3} x^{3 \alpha+2}}{\Gamma(3 \alpha+3)}-\frac{2 h^{2} x^{2 \alpha+2}}{\Gamma(2 \alpha+3)}-\frac{t h^{2} x^{2 \alpha}}{\Gamma(2 \alpha+1)} \\
& +\frac{2 h(h+2) x^{\alpha+2}}{\Gamma(\alpha+3)}-\frac{(1-t) h(h+2) x^{\alpha}}{\Gamma(\alpha+1)},
\end{aligned}
$$

Figure 2: when $\alpha=2, t=1$, the exact solution of example(4.3) and Elzaki transformation

\section{References}

[1] M. Caputo, The Green function of the diffusion of fluids in porous media with memory, Rend. Fis. Acc. Lincei (Ser. 9) 7 (1996) 243"C250.

[2] M. Giona, H.E. Roman, A theory of transport phenomena in disordered systems, Chem. Eng. J. 49 (1992) 1"C10.

[3] R. Hilfer, Exact solutions for a class of fractal time random walks, Fractals 3 (1995) 211"C216.

[4] F. Mainardi, Fractional diffusive waves in viscoelastic solids, in: J.L. Wagnern, F.R. Norwood (Eds.), IUTAM Symposium; ${ }^{2}$ Nonlinear Waves in Solids, ASME/AMR, Fairfield NJ, 1995, pp. 93"C97.

[5] S. Momani, Analytic and approximate solutions of space and time fractional telegraph equations, Appl. Math. Comput. 170 (2005) $1126 " \mathrm{C} 1134$.

[6] A. Yildirim, He's homotopy perturbation method for solving the space- and time-fractional telegraph equations, Int. J. Comput. Math. 87 (13) (2010) 2998"C3006.

[7] J. Chen, F. Liu, V. Anh, Analytical solution for the time-fractional telegraph equation by the method of separating variables, J. Math. Anal. Appl. 338(2008) 1364"C1377.

[8] F. Huang, Analytical Solution for the Time-Fractional Telegraph Equation, J. Appl. Math. (2009) 9, http://dx.doi.org/10.1155/2009/890158. Article ID 890158. 
[9] J. Biazar, M. Eslami, Analytic solution for telegraph equation by differential transform method, Phys. Lett. A 374 (29) (2010) 2904"C2906.

[10] J. Sunil, A new analytical modelling for fractional telegraph equation via Laplace transform, J. Appl. Math. 38 (2014) $3154 "$ C3163.

[11] S.J. Liao, The proposed homotopy analysis technique for the solution of nonlinear problems, (Ph. D) thesis, Shanghai Jiao Tong University, 1992.

[12] S.J. Liao, Beyond Perturbation: Introduction to the Homotopy Analysis Method, CRC Press, Chapman and Hall, Boca Raton, 2003.

[13] S. J. Liao, On the homotopy analysis method for nonlinear problems, Appl. Math. Comput. 147(2004):499-513.

[14] S.J. Liao, Homotopy analysis method: a new analytical technique for nonlinear problems, Commun. Nonlinear Sci. Numer. Simul. 2 (1997) 95“C100.

[15] Tarig. M. Elzaki, The New Integral Transform "ELzaki Transform”, J. Appl. Math. 0973-1768 Volume 7, Number 1 (2011), pp. 57"C64.

[16] I. Podlubny, Fractional Differential Equations, Academic, New York, 1999.

[17] S. J. Liao, Compatison between the homotopy analysis method and homotopy perturbation method, Appl. Math. Comput. 169(2005):1186-1194

[18] R. Garra, Analytic solution of a class of fractional differential equations with variable coefficients by operatorial methods, Int. J. nonlin. Sci. Numer. Simulat. 17(2012):1549-1554

[19] A. M. Wazwaz, The combined Laplace transform-Adomian decomposition method for handling nonlinear Volterra integro-differential equations, Appl. Math. Comput. 216(2010):1304-1309

[20] M. Khan, M. A. Gondal, S. Kumar, A new analytical procedure for nonlinear integral equation, Math. Comput. Modell. 55(2012):1892-1897

[21] S. Kumar, A. Yildirim, Y. Khan, L. Wei, A fractional model of the diffusion equation and its analytical solution using Laplace transform, Sci. Iran. B. 19(2012):1117-1123

[22] M. A. Gondal, M. Khan, Homotopy perturbation method for nonlinear exponential boundary Layer equation using Laplace transformation, Inter. J. Nonlinear Sci. Numer. Simul. 11(2010):1145-1153

[23] M. Madani, M. Fathizadeh, Y. Khan, A. Yildirim, On the coupling of the homotopy perturbation method and Laplace transformation, Math. Comput. Modell. 53(2011):1937-1945

[24] C. M. Chen, F. Liu, I. Turner. V. Anh, Y. Chen, Numerical approximation for a variable-order nonlinear reactionsubdiffusion equation, Numer. Algor. 63(2013):265-290

[25] J. Chen, F. Liu, K. Burrage, S. Shen, Numerical techniques for simulating a fractional mathematical model of epidermal wound healing, J. Appl. Math. Comput. 41(2013):33-47 [26] X. D. Zhang, J. Liu, J. Wen, B. Tang, Y. N. He, Analysis for one-dimensional time Tricomi-type equations by LDG methods, Numer. Algor. 63(2013):143-164

[27] X. D. Zhang, P. Z. Huang, X. L. Feng, L. L. Wei, Finite element method for two time-fractional tricomi-type equations, Numer. Meth. Part. Differ. Equ. 29(2013):1081-1096

[28] M. A. Khan, M. A. Gondal, I. Hussian, S. K. Vanani, A new comparaive study between homotopy analysis transform method and homotopy perturbation transform method on a semi-infinite domain, Math. Comput. Modell. 55(2012):11431150

[29] I. Hashim, O. Abdulaziz, S. Momani, Homotopy analysis method for fractional IVPs, Commun.Nonlinear Sci. Numer. Simul. 14(2009):674-684

[30] A. Mohebbi, M. Dehaghan, High order compact solution of the one dimensional linear hyperbolic equation, Numer. Meth. Part. Differ. Equ. 24(2008):1122-1135

[31] M. S. El-Azab, M. El-Glamel, A numerical algorithm for the solution of telegraph equation, Appl. Math. Comput. 190(2007):757-764

[32] S. J. Liao, On the homotopy analysis method for nonlinear problems, Appl. Math. Comput. 147(2004)499-513.

[33] J. Biazar, M. Eslami, Analytic solution for telegraph equation by differential transform method, Phys. Lett. A. 29(2010):2904-2906 